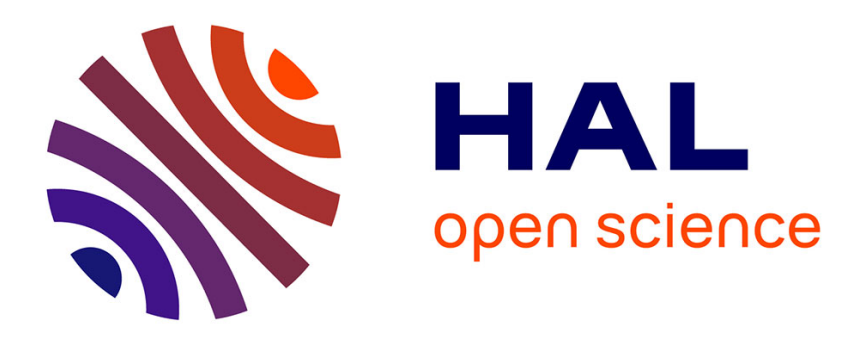

\title{
Régularisation spectrale et propriétés métriques des moyennes mobiles.
}

\author{
Mikhail Lifshits, Michel Weber
}

\section{To cite this version:}

Mikhail Lifshits, Michel Weber. Régularisation spectrale et propriétés métriques des moyennes mobiles.. Journal d'analyse mathématique, 2003, 89 (1), pp.1-14. 10.1007/BF02893074 . hal-00129651

\section{HAL Id: hal-00129651 \\ https://hal.science/hal-00129651}

Submitted on 8 Feb 2007

HAL is a multi-disciplinary open access archive for the deposit and dissemination of scientific research documents, whether they are published or not. The documents may come from teaching and research institutions in France or abroad, or from public or private research centers.
L'archive ouverte pluridisciplinaire HAL, est destinée au dépôt et à la diffusion de documents scientifiques de niveau recherche, publiés ou non, émanant des établissements d'enseignement et de recherche français ou étrangers, des laboratoires publics ou privés. 
Juin 2000

\title{
Régularisation Spectrale et
}

\section{Proprietés Métriques des Moyennes Mobiles}

\author{
Mikhail LIFSHITS et Michel WEBER \\ Université de St-Petersbourg et Université de Strasbourg
}

\begin{abstract}
We develop a spectral regularization technique for moving averages $B_{n}^{U, \phi}=\frac{1}{n} \sum_{j=\phi(n)}^{\phi(n)+n-1} U^{j}$, where $\phi$ is a nondecreasing map and $U: H \rightarrow H$ is a contraction of a Hilbert space $(H,\|\cdot\|)$. We obtain a spectral regularization inequality which allows to evaluate efficiently the increments $\left\|B_{m}^{U, \phi}(f)-B_{n}^{U, \phi}(f)\right\|, f \in H$, by means of $\hat{\mu}\left[\frac{1}{m}, \frac{1}{n}\right]$ where $\hat{\mu}$ is a properly regularized version of the spectral measure of $f$ with respect to $U$. We apply this inequality to investigation of metric properties of the sets of moving averages $\left\{B_{n}^{U, \phi}(f), n \in \mathcal{N}\right\}$ with fixed $f \in H$ and $\mathcal{N} \subset \mathbf{N}$. In particular, we obtain estimates of the associated covering numbers as well as of the related Littlewood-Paley-type square functions. This work extends our previous results concerning the case of classical averages $(\phi(n)=0)$. Since it is well known that the structure of general moving averages is more complicated, there is no surprise that the general results we obtain are sometimes less complete than their classical counterparts, and need suitable moment assumptions on the spectral measure (depending on the growth of the shift function $\phi)$. Nevertheless, when applied to classical situation, our estimates still yield optimal bounds.
\end{abstract}

AMS Subject Classification 1985: Primary 60F99, Secondary 28D99, 60G10, 60G12.

Keywords: spectral regularization, moving averages, entropy numbers, Littlewood-Paley square function.

First author supported by Russian Foundation for Basic Research, grant 99-01-00112, and INTAS grant 99-01317. 


\section{Introduction}

Soient $(H,\|\cdot\|)$ un espace de Hilbert et considérons une contraction arbitraire $U: H \rightarrow H$. Soit aussi $\phi: \mathbf{R}_{+} \rightarrow \mathbf{R}_{+}$une fonction croissante, dérivable et telle que $\phi(\mathbf{N}) \subset \mathbf{N}$. Considérons alors la suite de moyennes mobiles

$$
\forall n \geq 1, \quad B_{n}^{U, \phi}=B_{n}^{\phi}=\frac{1}{n} \sum_{j=\phi(n)}^{\phi(n)+n-1} U^{j}
$$

Lorsque $\phi(n)=0$ pour tout $n \in \mathbf{N}$, nous utiliserons la notation standard

$$
A_{n}=B_{n}^{\phi}=\frac{1}{n} \sum_{j=0}^{n-1} U^{j}
$$

Dans un travail récent $[\mathbf{L W 1}]$, [LW2], nous avions montré comment étudier efficacement la structure de l'ensemble $A(f)=\left\{A_{n}(f), n \geq 1\right\}, f \in H$, à l'aide d'une méthode de régularisation spectrale. Le principe de cette méthode est contenu dans une inégalité que nous rappelons. Soit $f \in H$ et notons $\mu$ la mesure spectrale de $f$ relativement à $U$. Nous régularisons cette mesure en une mesure $\hat{\mu}$ définie de la façon suivante:

$$
\hat{\mu}(d y)=\int\left\{\frac{|\theta|^{2}}{|y|^{3}} \wedge \frac{1}{|\theta|}\right\} \mu(d \theta) d y, \quad y \in[-\pi, \pi) .
$$

Alors ([LW], Théorème 1) pour tous entiers positifs $m \geq n$

$$
\left\|A_{m}(f)-A_{n}(f)\right\|_{2, \mu}^{2} \leq 4 \pi \hat{\mu}\left(\frac{1}{m}, \frac{1}{n}\right] .
$$

Nous avons montré l'efficacité remarquable de cette inégalité en retrouvant de façon très simple des estimations optimales de l'entropie métrique de l'ensemble $A(f)$ et de la fonction carré de Littlewood-Paley. En effet, soit $N(A(f), \varepsilon)$ le cardinal minimal d'un recouvrement de $A(f)$ par des boules hilbertiennes de rayon $\varepsilon$ centrées dans $A(f)$. L'estimation universelle de Talagrand $[\mathbf{T}]$

$$
N(A(f), \varepsilon) \leq 6 \pi\|f\|^{2} \varepsilon^{-2}+1
$$

se déduit facilement de (1.2). D'autre part, il résulte immédiatement de (1.2) que pour toute suite croissante $\left(n_{p}\right)$ d'entiers positifs

$$
\sum_{p=1}^{\infty}\left\|A_{n_{p+1}}(f)-A_{n_{p}}(f)\right\|^{2} \leq 6 \pi\|f\|^{2} .
$$

On retrouve donc ainsi un résultat récent de Jones, Ostrovskii et Rosenblatt [JOR].

Nous proposons dans ce travail de développer une approche similaire pour l'étude des moyennes mobiles $B_{n}^{\phi}$ en donnant une construction propre de la mesure régularisée, 
similaire à (1.1), et permettant d'obtenir des analogues de (1.3) et (1.4) pour les moyennes mobiles.

Il importe cependant ici de souligner les considérables différences de nature qui se présentent lorsqu'on passe de l'étude des moyennes classiques à celles des moyennes mobiles. Alors que les moyennes classiques montrent des caractéristiques très voisines des martingales dyadiques (voir $[\mathbf{J K R W}]$ et $[\mathbf{S}]$ ) et permettent donc différentes approches, les moyennes mobiles s'en éloignent quant à elles fortement. Il résulte du travail présenté dans $[\mathbf{B J R}]$, ainsi que de travaux antérieurs (voir aussi [BJR] pour des références) que les moyennes mobiles présentent un comportement nettement plus complexe, surtout en ce qui concerne leur convergence presque sûre.

Dans le paragraphe qui suit, nous établissons une inégalité de régularisation spectrale pour les moyennes mobiles.

\section{L'inégalité de régularisation spectrale}

Introduisons les noyaux spectraux associés aux moyennes classiques et aux moyennes mobiles. Dans le cas des moyennes classiques, on considère la suite de noyaux spectraux suivante $\left\{V_{n}(\theta)=\frac{e^{i n \theta}-1}{n\left(e^{i \theta}-1\right)}\right\}$. Pour les moyennes mobiles, nous aurons à considérer la suite de noyaux spectraux $\left\{W_{n}(\theta)=e^{i \phi(n) \theta} V_{n}(\theta)\right\}$. Rappelons quelques proprietés élémentaires des noyaux $V_{n}$ et $W_{n}$. Par définition, pour tous entiers positifs $n, m$,

$$
\left|W_{m}(\theta)-W_{n}(\theta)\right| \leq\left|V_{m}(\theta)-V_{n}(\theta)\right|+\left|e^{i(\phi(m)-\phi(n)) \theta}-1\right|\left|V_{m}(\theta)\right| .
$$

Concernant la première différence (qui a été d'ailleurs estimée dans [LW2]) on a, pour tout $|\theta| \leq \pi$

$$
\left|V_{m}(\theta)-V_{n}(\theta)\right| \leq \frac{2(m-n)}{m} \wedge \frac{\pi}{n|\theta|} \wedge \frac{\pi}{4}(m-n)|\theta| .
$$

Concernant la seconde différence, on a

$$
\left|e^{i(\phi(m)-\phi(n)) \theta}-1\right| \leq|\theta|(\phi(m)-\phi(n)) \wedge 1
$$

et $\left|V_{m}(\theta)\right| \leq 1$. Il s'en suit

$$
\left|W_{m}(\theta)-W_{n}(\theta)\right|^{2} \leq 2\left|V_{m}(\theta)-V_{n}(\theta)\right|^{2}+2\left\{|\theta|^{2}(\phi(m)-\phi(n))^{2} \wedge 1\right\}\left\{\frac{\pi^{2}}{|\theta|^{2} m^{2}} \wedge 1\right\} .
$$

Introduisons en suivant $[\mathbf{W}]$, le noyau

$$
Q(\theta, y)=\frac{1}{|\theta|} \wedge \frac{|\theta|}{y^{2}}
$$

pour $\theta \in[-\pi, \pi), y \in(0,1]$. Dans les Lemmes 1 et 2 qui suivent, nous allons utiliser ce noyau pour évaluer efficacement les deux parties du membre à droite de (2.2). Ce noyau est un peu différent de celui employé dans [LW2] ( $c f$. l'intégrand dans (1.1)) mais il implique toutes estimations nécessaires de façon encore plus simple. 
Lemme 1. Soit $m \geq n$ deux entiers positifs. Alors pour tout $\theta \in[-\pi, \pi)$

$$
2 \pi \int_{1 / m}^{1 / n} Q(\theta, y) d y+4 \mathbf{1}_{\left[\frac{1}{m}, \frac{1}{n}\right)}(|\theta|) \geq\left|V_{m}(\theta)-V_{n}(\theta)\right|^{2}
$$

Démonstration. Considérons trois cas:

1) $|\theta| \geq \frac{1}{n}$. Par définition de $Q$ et par (2.1)

$$
\int_{1 / m}^{1 / n} Q(\theta, y) d y=\int_{1 / m}^{1 / n} \frac{1}{|\theta|} d y=\frac{m-n}{m} \frac{1}{n|\theta|} \geq \frac{1}{2 \pi}\left|V_{m}(\theta)-V_{n}(\theta)\right|^{2} .
$$

2) $|\theta| \leq \frac{1}{m}$. Alors pour les mêmes raisons

$$
\int_{1 / m}^{1 / n} Q(\theta, y) d y=\int_{1 / m}^{1 / n} \frac{|\theta|}{y^{2}} d y=(m-n)|\theta| \geq(m-n)^{2}|\theta|^{2} \wedge 1 \geq \frac{1}{4}\left|V_{m}(\theta)-V_{n}(\theta)\right|^{2} .
$$

3) $\frac{1}{n}>|\theta| \geq \frac{1}{m}$. Ce cas est évident: à l'aide de (2.1), nous avons $\left|V_{m}(\theta)-V_{n}(\theta)\right| \leq 2$.

Lemme 2. Soit $m \geq n$ deux entiers positifs. Alors pour tout $\theta \in[-\pi, \pi)$,

$$
\int_{1 / m}^{1 / n} \phi^{\prime}(1 / y) Q(\theta, y) d y \geq\left\{|\theta|^{2}(\phi(m)-\phi(n))^{2} \wedge 1\right\}\left\{\frac{1}{|\theta|^{2} m^{2}} \wedge 1\right\} \text {. }
$$

Démonstration. Considérons deux cas:

1) $|\theta| \geq \frac{1}{m}$. Alors

$$
\begin{gathered}
\int_{1 / m}^{1 / n} \phi^{\prime}(1 / y)\left\{\frac{1}{|\theta|} \wedge \frac{|\theta|}{y^{2}}\right\} d y=\int_{n}^{m} \phi^{\prime}(u)\left\{\frac{1}{|\theta| u^{2}} \wedge|\theta|\right\} d u \geq \\
\int_{n}^{m} \phi^{\prime}(u)\left\{\frac{1}{|\theta| m^{2}} \wedge|\theta|\right\} d u=\frac{\phi(m)-\phi(n)}{|\theta| m^{2}}=\frac{(\phi(m)-\phi(n))|\theta|}{|\theta|^{2} m^{2}} .
\end{gathered}
$$

En utilisant l'inégalité élémentaire $x \geq x^{2} \wedge 1$ avec $x=(\phi(m)-\phi(n))|\theta|$ nous obtenons le résultat de Lemme 2 .

2) $|\theta| \leq \frac{1}{m}$. Alors

$$
\begin{gathered}
\int_{1 / m}^{1 / n} Q(\theta, y) d y=\int_{1 / m}^{1 / n} \phi^{\prime}(1 / y) \frac{|\theta|}{y^{2}} d y= \\
|\theta| \int_{n}^{m} \phi^{\prime}(u) d u=|\theta|(\phi(m)-\phi(n)) .
\end{gathered}
$$

A l'aide de la même inégalité élémentaire, on a

$$
|\theta|(\phi(m)-\phi(n)) \geq|\theta|^{2}(\phi(m)-\phi(n))^{2} \wedge 1,
$$

et le Lemme 2 est donc complètement démontré . 
On déduit de (2.2), du Lemme 1 et du Lemme 2 que

$$
\left|W_{m}(\theta)-W_{n}(\theta)\right|^{2} \leq \int_{1 / m}^{1 / n}\left(4 \pi+2 \pi^{2} \phi^{\prime}(1 / y)\right) Q(\theta, y) d y+8 \mathbf{1}_{\left[\frac{1}{m}, \frac{1}{n}\right)}(|\theta|) .
$$

Soit maintenant $\mu$ une mesure arbitraire sur $[-\pi, \pi)$. Introduisons une nouvelle mesure $\hat{\mu}$, une régularisation de la mesure $\mu$, à l'aide de notre noyau $Q$ en posant

$$
\hat{\mu}(d y)=\int_{-\pi}^{\pi}\left(4 \pi+2 \pi^{2} \phi^{\prime}(1 / y)\right) Q(\theta, y) \mu(d \theta) d y+8 \mu(d y)
$$

En intégrant (2.5) par rapport à la measure $\mu$, on obtient

$$
\left\|W_{m}-W_{n}\right\|_{2, \mu}^{2} \leq \hat{\mu}\left(\frac{1}{m}, \frac{1}{n}\right]
$$

\section{Estimations des moyennes mobiles}

Soit $\left\{B_{n}^{\phi}\right\}$ un système de moyennes mobiles associés à une contraction arbitraire $U$ de l'espace de Hilbert $H$. Le lemme spectral pour les contractions (voir $[\mathbf{K}]$, p.9495) permet de ramener l'étude des opérateurs polynômiaux de variable $U$, à l'étude de polynômes trigonométriques dans l'espace $L^{2}([-\pi, \pi), \mu)$ pour une mesure $\mu$ appropriée. Plus précisement, pour tout élément $f \in H$, il existe une mesure $\mu_{f}$ sur $[-\pi, \pi)$ telle que $\mu_{f}[-\pi, \pi)=\|f\|^{2}$ et pour tout polynôme $\mathcal{P}$

$$
\|\mathcal{P}(U)(f)\| \leq\left\|\mathcal{P}\left(e^{i \cdot}\right)\right\|_{2, \mu}
$$

La mesure $\mu_{f}$ est la mesure spectrale associée à $f$. En posant $\mathcal{P}\left(e^{i \theta}\right)=W_{m}(\theta)-W_{n}(\theta)$, on obtient

$$
\left\|B_{m}^{\phi}(f)-B_{n}^{\phi}(f)\right\| \leq\left\|W_{m}-W_{n}\right\|_{2, \mu_{f}}
$$

Nous pouvons maintenant énoncer notre inégalité de base.

Théorème 1. Soit $\left\{B_{n}^{\phi}\right\}$ un système de moyennes mobiles associés à une contraction arbitraire $U$ d'un espace de Hilbert $H$. Soit $\mu$ la mesure spectrale d'un élément $f \in H$. Soit $\hat{\mu}$ la mesure régularisée définie dans (2.6). Alors pour tous $m \geq n$ positifs

$$
\left\|B_{m}^{\phi}(f)-B_{n}^{\phi}(f)\right\| \leq \hat{\mu}\left(\frac{1}{m}, \frac{1}{n}\right] .
$$

Démonstration: Il suffit de joindre (2.7) à (3.1). 
Corollaire 1. (Une borne pour la fonction carré de Littlewood-Paley)

$$
\Phi(\theta)=\frac{1}{|\theta|} \int_{\frac{1}{|\theta|} \vee 1}^{\infty} \frac{\phi^{\prime}(u)}{u^{2}} d u+\phi\left(|\theta|^{-1}\right)|\theta| \mathbf{1}_{\{|\theta| \leq 1\}}
$$

Alors pour tout $f \in H$ de mesure spectrale $\mu$, et pour toute suite croissante d'entiers positifs $\left(n_{p}\right)$

$$
\sum_{p=1}^{\infty}\left\|B_{n_{p+1}}^{\phi}(f)-B_{n_{p}}^{\phi}(f)\right\|^{2} \leq 2 \pi^{2} \int \Phi(\theta) \mu(d \theta)+(4 \pi+8) \mu[-\pi, \pi) .
$$

Démonstration. En utilisant (3.2) et (2.6) on obtient

$$
\begin{aligned}
\sum_{p=1}^{\infty}\left\|B_{n_{p+1}}^{\phi}(f)-B_{n_{p}}^{\phi}(f)\right\|^{2} & \leq \sum_{p=1}^{\infty} \hat{\mu}\left(\frac{1}{n_{p+1}}, \frac{1}{n_{p}}\right]=\hat{\mu}\left(0, \frac{1}{n_{1}}\right] \\
& \leq \int_{0}^{1} d y \int_{-\pi}^{\pi}\left(4 \pi+2 \pi^{2} \phi^{\prime}(1 / y)\right) Q(\theta, y) \mu(d \theta)+8 \mu(0,1] \\
& \leq(4 \pi+8) \mu[-\pi, \pi)+2 \pi^{2} \int_{-\pi}^{\pi} \int_{0}^{1} \phi^{\prime}(1 / y)\left\{\frac{1}{|\theta|} \wedge \frac{|\theta|}{y^{2}}\right\} d y \mu(d \theta) .
\end{aligned}
$$

En effectuant en outre pour chaque $\theta$ le changement de variables $y=u^{-1}$, on obtient

$$
\begin{aligned}
\int_{0}^{1} \phi^{\prime}(1 / y)\left\{\frac{1}{|\theta|} \wedge \frac{|\theta|}{y^{2}}\right\} d y & \leq \int_{0}^{|\theta| \wedge 1} \frac{\phi^{\prime}(1 / y)}{|\theta|} d y+\int_{|\theta| \wedge 1}^{1} \phi^{\prime}(1 / y) \frac{|\theta|}{y^{2}} d y \\
& \leq \frac{1}{|\theta|} \int_{\frac{1}{|\theta|} \vee 1}^{\infty} \frac{\phi^{\prime}(u)}{u^{2}} d u+\phi\left(|\theta|^{-1}\right)|\theta| \mathbf{1}_{\{|\theta| \leq 1\}}=\Phi(\theta) .
\end{aligned}
$$

Remarque. Pour des fonctions $\phi$ à croissance sous-linéaire et satisfaisant l'inégalité $\sup _{u} \phi^{\prime}(u) \leq C$, l'expression de $\Phi$ donnée en (3.3) est uniformément bornée; et donc $\sum_{p=1}^{\infty}\left\|B_{n_{p+1}}^{\phi}(f)-B_{n_{p}}^{\phi}(f)\right\|^{2} \leq\left(2 \pi^{2} \sup _{\theta} \Phi(\theta)+4 \pi+8\right) \mu[-\pi, \pi) \leq\left(4 \pi^{2} C+4 \pi+8\right)\|f\|^{2}$.

Une estimation uniforme semblable a été établie pour les moyennes classiques $(\phi(u)=0$; voir (1.4)), cf aussi [JOR] ou [LW2].

L'estimation (3.4) reste encore raisonnable pour des fonctions $\phi$ de croissance surlinéaire, mais seulement pour des éléments $f$ pour lequels $\int \Phi(\theta) \mu(d \theta)<\infty$. Si par exemple, $\phi(u) \approx u^{\alpha}, \alpha \in[1,2)$, l'estimation (3.4) est efficace sous l'hypothèse spectrale:

$$
\int|\theta|^{1-\alpha} \mu(d \theta)<\infty
$$


Si $\alpha \geq 2$, alors $\Phi(\theta)$ est infini et l'estimation (3.4) cesse d'être efficace.

Nous allons néanmoins montrer plus loin (voir Théorème 3) que l'estimateur (3.4) est vraiment optimal. Lorsque la suite $\left(n_{p}\right)$ est à croissance très rapide, on peut obtenir plus. Dans cette direction, introduisons l'hypothèse suivante relative à la suite $\left(n_{p}\right)$ :

$$
M_{1}=\sup _{l \geq 1} \sum_{p=l}^{\infty}\left[\frac{n_{l}}{n_{p}}\right]^{2}<\infty
$$

L'énoncé équivalent à $(G 1)$ est

$$
\exists q \in \mathbf{N}, c>0 \quad: \quad \forall p \quad(1+c) n_{p} \leq n_{p+q}
$$

Nous considérons aussi une hypothèse encore plus forte:

$$
\exists q \in \mathbf{N}, c>0 \quad: \quad \forall p \quad \max \left\{\phi\left(n_{p}\right) ;(1+c) n_{p}\right\} \leq n_{p+q} .
$$

Cette hypothèse est vérifiée si par exemple $\left(n_{p}\right)$ est une suite du type exponentielle double, à savoir $n_{p} \sim \exp \left\{C a^{p}\right\}$ et des fonctions $\phi$ polynômiales, $\phi(u) \sim u^{\alpha}$.

Les estimations que nous obtenons, feront intervenir la fonction inverse suivante de la suite $\left(n_{p}\right)$,

$$
L(x)= \begin{cases}\sup \left\{p: n_{p} \leq x\right\}, & \text { si } x \geq n_{1} \\ 0, & \text { si } x<n_{1}\end{cases}
$$

Proposition 2. Supposons que la suite $\left(n_{p}\right)$ satisfasse l'hypothèse (G1). Alors, pour tout élément $f \in H$

$$
\sum_{p=1}^{\infty}\left\|B_{n_{p+1}}^{\phi}(f)-B_{n_{p}}^{\phi}(f)\right\|^{2} \leq 2\left(6 \pi+\pi^{2} M_{1}\right)\|f\|^{2}+2 I\left(\mu_{f}\right),
$$

où

$$
I\left(\mu_{f}\right)=\int\left[L\left(|\theta|^{-1}\right)-L\left(\phi^{-1}\left(|\theta|^{-1}\right)\right)\right]_{+} \mu_{f}(d \theta) .
$$

Si de plus l'hypothèse (G2) est vérifiée, alors nous avons aussi

$$
\sum_{p=1}^{\infty}\left\|B_{n_{p+1}}^{\phi}(f)-B_{n_{p}}^{\phi}(f)\right\|^{2} \leq 2\left(6 \pi+\pi^{2} M_{1}+q+1\right)\|f\|^{2} .
$$

Démonstration: A l'aide de (3.1) et (2.2) on a

$$
\sum_{p=1}^{\infty}\left\|B_{n_{p+1}}^{\phi}(f)-B_{n_{p}}^{\phi}(f)\right\|^{2} \leq 2 \sum_{p=1}^{\infty} \int\left|V_{n_{p+1}}(\theta)-V_{n_{p}}(\theta)\right|^{2} \mu_{f}(d \theta)+
$$




$$
2 \int\left[\sum_{p=1}^{\infty}\left\{|\theta|^{2}\left(\phi\left(n_{p+1}\right)-\phi\left(n_{p}\right)\right)^{2} \wedge 1\right\}\left\{\frac{\pi^{2}}{|\theta|^{2} n_{p+1}^{2}} \wedge 1\right\}\right] \mu_{f}(d \theta) .
$$

D'après $[\mathbf{L W 2}]$, le premier terme peut se majorer par $12 \pi\|f\|^{2}$. Considérons le second terme. Fixons $\theta$, et posons $p^{-}=L\left(\phi^{-1}\left(|\theta|^{-1}\right)\right)-1, p^{*}=L\left(|\theta|^{-1}\right)$. Alors

$$
\sum_{p=1}^{p^{-}}\left(\phi\left(n_{p+1}\right)-\phi\left(n_{p}\right)\right)^{2} \leq \sum_{p=1}^{p^{-}}\left(\phi\left(n_{p+1}\right)-\phi\left(n_{p}\right)\right) \phi\left(n_{p^{-}+1}\right) \leq \phi\left(n_{p^{-}+1}\right)^{2} \leq|\theta|^{-2}
$$

De la même manière, $(G 1)$ implique

$$
\sum_{p=p^{*}}^{\infty} \frac{1}{n_{p+1}^{2}} \leq \frac{M_{1}}{n_{p^{*}+1}^{2}} \leq M_{1}|\theta|^{2}
$$

Il s'en suit que le second intégrand dans (3.6) est majoré par

$$
1+\pi^{2} M_{1}+\sum_{p=1}^{\infty} \mathbf{1}_{\left\{p^{-}<p<p^{*}\right\}}=1+\pi^{2} M_{1}+\left[L\left(|\theta|^{-1}\right)-L\left(\phi^{-1}\left(|\theta|^{-1}\right)\right)\right]_{+},
$$

d'où par intégration, la première partie de la Proposition.

Supposons maintenant que la condition $(G 2)$ soit vérifiée. La croissance des fonctions $L$ et $\phi$ montrent que pour chaque $u$,

$$
L(u)-L\left(\phi^{-1}(u)\right) \leq q+1
$$

D'où $I\left(\mu_{f}\right) \leq(q+1) \mu_{f}(-\pi, \pi]=(q+1)\|f\|^{2}$. La deuxième assertion de la Proposition se déduit alors du résultat précédemment établi.

Remarque. Dans le cas $n_{p} \sim e^{a p}, \phi(u) \sim u^{\alpha}$, nous avons $L(x) \sim \frac{\log x}{a}$ de sorte que

$$
L\left(|\theta|^{-1}\right)-L\left(\phi^{-1}\left(|\theta|^{-1}\right)\right) \sim c|\log \theta|, \quad c=\frac{\alpha-1}{a \alpha} .
$$

On observe que notre estimateur est fini si $I\left(\mu_{f}\right)<\infty$, c'est à dire si,

$$
\int|\log \theta| \mu_{f}(d \theta)<\infty
$$

ce que est beaucoup moins restrictif que (3.5), et améliore aussi un résultat récemment publié de Gamet-Weber [GW, Théorème 1.2].

L'énoncé suivant concerne des suites arbitraires $\left(n_{p}\right)$, mais s'applique essentiellement aux éléments $f$ de $H$ dont le mesure spectrale charge très faiblement le voisinage de zéro. 
Proposition 3. Pour toute suite $\left(n_{p}\right)$ et tout élément $f \in H$, on a

$$
\sum_{p=1}^{\infty}\left\|B_{n_{p+1}}^{\phi}(f)-B_{n_{p}}^{\phi}(f)\right\|^{2} \leq 12 \pi\|f\|^{2}+\left(\frac{\pi^{4}}{3}+2\right) \int \frac{\mu_{f}(d \theta)}{|\theta|} .
$$

Démonstration. On répète le raisonnement précédent, sauf qu'en l'absence de l'hypothèse $(G 1)$ on utilise en lieu et place de (3.9), l'estimateur trivial suivant

$$
\sum_{p=p^{*}}^{\infty} \frac{1}{n_{p+1}^{2}} \leq \sum_{n=n_{p^{*}+1}}^{\infty} \frac{1}{n^{2}} \leq \frac{\pi^{2}}{6} \frac{1}{n_{p^{*}+1}^{2}} \leq \frac{\pi^{2}}{6}|\theta| .
$$

Comme en outre $L\left(|\theta|^{-1}\right) \leq|\theta|^{-1}$, on a

$$
I\left(\mu_{f}\right) \leq \int \frac{\mu_{f}(d \theta)}{|\theta|}
$$

On obtient alors (3.10) de la même manière que (3.6), par intégration.

Nous allons à présent montrer l'optimalité de la majoration donnée en (3.4), en établissant une minoration du même ordre de la fonction carré de Littlewood-Paley associée aux moyennes mobiles.

Théorème 3. Soit $U$ un operateur unitaire. Soit $\phi(\cdot)$ une fonction à variation régulière de l'ordre $\alpha \in(1,2)$, et supposons que la fonction $\phi(u) / u$ soit croissante. Il existe alors une constante $c=c(\phi)$, et une suite croissante d'entiers positifs $\left(n_{p}\right)$, telles que pour tout élément $f \in H$, l'on ait:

$$
\sum_{p=1}^{\infty}\left\|B_{n_{p+1}}^{\phi}(f)-B_{n_{p}}^{\phi}(f)\right\|^{2} \geq c \int \Phi(\theta) \mu_{f}(d \theta) .
$$

Démonstration. Rappelons que dans le cas des opérateurs unitaires, le lemme spectral est une égalité spectrale; de sorte que l'on a:

$$
\sum_{p=1}^{\infty}\left\|B_{n_{p+1}}^{\phi}(f)-B_{n_{p}}^{\phi}(f)\right\|^{2}=\int \sum_{p=1}^{\infty}\left|W_{n_{p+1}}(\theta)-W_{n_{p}}(\theta)\right|^{2} \mu_{f}(d \theta) .
$$

Il suffira par conséquent d'établir l'estimation suivante:

$$
\sum_{p=1}^{\infty}\left|W_{n_{p+1}}(\theta)-W_{n_{p}}(\theta)\right|^{2} \geq c \Phi(\theta)
$$

La construction de la suite critique $\left(n_{p}\right)$ est remarquablement simple. Soit $\psi(\cdot)$ la fonction inverse de la fonction $u \rightarrow \phi(u) / u$. Alors $\psi$ est aussi une fonction à variation 
régulière d'ordre $\beta=\frac{1}{\alpha-1}$. Posons $n_{p}=[\psi(p)]$. Nous allons tout d'abord établir quelques propriétés concernant la suite $\left(n_{p}\right)$, utiles pour ce qui suivra. Introduisons à cet effet les deux fonctions à variation lente suivantes $g(u)=\frac{\phi(u)}{u^{\alpha}}$ et $h(p)=\frac{\psi(p)}{p^{\beta}}$. Alors

$$
\phi\left(n_{p}\right) \sim \phi(\psi(p))=\psi(p)^{\alpha} g(\psi(p))=p^{\beta \alpha} h(p)^{\alpha} g(\psi(p)) .
$$

Les propriétés classiques des fonctions à variation lente montrent que

$$
\begin{gathered}
\phi\left(n_{p+1}\right)-\phi\left(n_{p}\right) \sim \phi(\psi(p+1))-\phi(\psi(p)) \sim \beta \alpha p^{\beta \alpha-1} h(p)^{\alpha} g(\psi(p))= \\
\beta \alpha p^{\beta \alpha-1}\left[\frac{\psi(p)}{p^{\beta}}\right]^{\alpha} \frac{\phi(\psi(p))}{\psi(p)^{\alpha}}=\beta \alpha p^{-1} \phi(\psi(p))=\beta \alpha \psi(p) \sim \beta \alpha n_{p} .
\end{gathered}
$$

Etablissons à présent (3.11). Remarquons tout d'abord que

$$
W_{n_{p+1}}(\theta)-W_{n_{p}}(\theta)=\left(e^{i \phi\left(n_{p+1}\right) \theta}-e^{i \phi\left(n_{p}\right) \theta}\right) V_{n_{p+1}}(\theta)+e^{i \phi\left(n_{p}\right) \theta}\left(V_{n_{p+1}}(\theta)-V_{n_{p}}(\theta)\right) .
$$

Ceci implique

$$
\left|W_{n_{p+1}}(\theta)-W_{n_{p}}(\theta)\right| \geq\left|e^{i\left(\phi\left(n_{p+1}\right)-\phi\left(n_{p}\right)\right) \theta}-1\right| \cdot\left|V_{n_{p+1}}(\theta)\right|-\left|V_{n_{p+1}}(\theta)-V_{n_{p}}(\theta)\right| .
$$

En invoquant (2.1), on en déduit que

$$
\sup _{\theta}\left|V_{n_{p+1}}(\theta)-V_{n_{p}}(\theta)\right| \leq \frac{2\left(n_{p+1}-n_{p}\right)}{n_{p}} \leq \frac{2(\psi(p+1)-\psi(p)+1)}{\psi(p+1)} \rightarrow 0 \quad(p \rightarrow \infty) .
$$

Il est donc naturel d'examiner les paires $(p, \theta)$ pour lesquelles le premier terme de (3.13) n'est pas asympotiquement petit. Remarquons alors tout d'abord qu'il existe un nombre réel $a>0$ tel que $\frac{a}{2 \pi}$ et $\frac{\beta \alpha a}{2 \pi}$ ne sont pas entiers. On peut donc déterminer un petit voisinage $\Delta$ de zéro, tel que

$$
\{(a+\Delta) \cup \beta \alpha(a+2 \Delta)\} \bigcap 2 \pi \mathbf{Z}=\emptyset .
$$

Soit

$$
c_{1}=\inf _{u \in a+\Delta}\left|e^{i u}-1\right|, \quad c_{2}=\inf _{u \in \alpha \beta a+2 \Delta}\left|e^{i u}-1\right|, \quad c_{3}=\sup _{u \in a+\Delta}|u| .
$$

Considérons une paire $(\theta, p)$ vérifiant

$$
|\theta| n_{p+1} \in a+\Delta
$$

Alors

$$
\left|V_{n_{p+1}}(\theta)\right|=\frac{\left|e^{i n_{p+1}}-1\right|}{n_{p+1}\left|e^{i \theta}-1\right|} \geq \frac{c_{1}}{n_{p+1}|\theta|} \geq \frac{c_{1}}{c_{3}} .
$$

D'autre part, en vertu de (3.12), on a pour tout $p$ suffisamment grand,

$$
\phi\left(n_{p+1}\right)-\phi\left(n_{p}\right) \sim \beta \alpha n_{p} \sim \beta \alpha n_{p+1} .
$$


On en déduit donc que pour tout entier $p$ suffisamment grand,

$$
\phi\left(n_{p+1}\right)-\phi\left(n_{p}\right)|\theta|=\beta \alpha n_{p+1}(1+o(1)) \in \beta \alpha(a+2 \Delta)
$$

et

$$
\left|e^{i\left(\phi\left(n_{p+1}\right)-\phi\left(n_{p}\right)\right) \theta}-1\right| \geq c_{2} .
$$

On déduit alors de (3.13), (3.15), et (3.16) que l'hypothèse (3.14) implique pour tout entier $p$ suffisamment grand

$$
\left|W_{n_{p+1}}(\theta)-W_{n_{p}}(\theta)\right| \geq \frac{c_{1} c_{2}}{2 c_{3}} .
$$

En outre, on peut choisir $\delta>0$ suffisamment petit pour que pout tout $\theta$ assez petit et tout entier $p \in\left[(1-\delta) \psi^{-1}(a /|\theta|),(1+\delta) \psi^{-1}(a /|\theta|)\right]$, l'on ait

$$
n_{p+1}|\theta| \sim \psi(p)|\theta| \in a+\Delta .
$$

Il existe donc au moins $2 \delta \psi^{-1}(a /|\theta|)-1$ solutions de (3.14). Rappelons que

$$
2 \delta \psi^{-1}(a /|\theta|)=2 \delta \phi(a /|\theta|) \frac{|\theta|}{a} \sim 2 \delta a^{\alpha-1} \phi\left(|\theta|^{-1}\right)|\theta|
$$

et que

$$
\Phi(\theta)=\phi\left(|\theta|^{-1}\right)|\theta|+\frac{1}{|\theta|} \int_{\frac{1}{|\theta|}}^{\infty} \frac{\phi^{\prime}(u)}{u^{2}} d u=\frac{1}{|\theta|} \int_{\frac{1}{|\theta|}}^{\infty} \frac{2 \phi(u)}{u^{3}} d u \sim|\theta| \phi\left(|\theta|^{-1}\right) .
$$

En combinant (3.17), (3.18) et (3.19) on obtient (3.11).

\section{Estimation de l'entropie métrique}

Soit $\mathcal{N}=\left(n_{p}\right)$ une sous-suite et $N(\mathcal{N}, f, \varepsilon)$ désigne le cardinal minimal d'un recouvrement dans $H$ de l'ensemble $\left\{B_{n_{p}}^{\phi}(f), p \geq 1\right\}$ par des boules de rayon $\varepsilon$. Dans certains cas nos résultats précédents permettent d'estimer ces nombres d'entropie des moyennes mobiles.

Proposition 4. Sous l'hypothèse $(G 1)$, pour tout $f \in H$ tel que $I\left(\mu_{f}\right)<\infty$, on a

$$
\forall \varepsilon>0 \quad N(\mathcal{N}, f, \varepsilon) \leq \frac{C}{\varepsilon^{2}}
$$

avec $C=2\left(6 \pi+\pi^{2} M_{1}\right)\|f\|^{2}+2 I\left(\mu_{f}\right)$. Si de plus la condition $(G 2)$ est verifiée, nous avons l'inégalité (4.1) avec $C=2\left(6 \pi+\pi^{2} M_{1}+q+1\right)\|f\|^{2}$.

Démonstration. Soit $f \in H$ et soit $\varepsilon>0$. Soient $t_{1}<\ldots<t_{r}$ une suite ordonnée de points de $\mathcal{N}$ telle que

$$
\forall i \neq j, \quad\left\|B_{t_{i}}^{\phi}(f)-B_{t_{j}}^{\phi}(f)\right\| \geq \varepsilon .
$$


Appliquons la Proposition 2 à la sous-suite

$$
\mathbf{N}^{\prime}=\left\{t_{1}, \ldots, t_{r}, n_{p_{o}+1}, n_{p_{o}+2}, \ldots\right\}
$$

si $t_{r}=n_{p_{o}}$. Comme la suite extraite $N^{\prime}$ vérifie à son tour l'hypothèse $(\mathrm{G} 1)$, on a

$$
\varepsilon^{2} r \leq \sum_{j=1}^{r-1}\left\|B_{t_{j+1}}^{\phi}(f)-B_{t_{j}}^{\phi}(f)\right\|^{2} \leq C .
$$

Et par conséquent,

$$
N(\mathcal{N}, f, \varepsilon) \leq \frac{C}{\varepsilon^{2}}
$$

D'où la première partie de la Proposition; la seconde partie de l'énoncé résulte du même argument.

\section{Références:}

[BJR] Bellow A., Jones R., Rosenblatt J., Convergence of moving averages (1990) Ergodic Theory and Dynamical Systems 10, p.43-62.

[GW] Gamet C., Weber M., (1999) Entropy numbers of some ergodic averages, Theory of Probability and Applications 44, p.776-795.

[JKRW] Jones R., Kaufman R., Rosenblatt J., Wierdl M., (1998) Oscillation in ergodic theory, Ergodic Theory Dynam. Systems 18 (1998), no. 4, p.889-935.

[JOR] Jones R., Ostrovskii I., Rosenblatt J., (1996) Square functions in ergodic theory, Ergodic Theory and Dynamical Systems, 22, p.267-305.

[K] Krengel U. (1985) Ergodic theorems, de Gruyter.

[LW1] Lifshits M., Weber M. (1996) Régularisation spectrale en théorie ergodique et en probabilités, Note aux Comptes Rendus Acad. Sci. Paris, 324 Série 1, p.99-103.

[LW2] Lifshits M., Weber M. (2000) Spectral regularization inequalities, Math. Scand. 86 p.75-99.

[S] Stroock D. (1993) Probability theory, an analytic view, Cambridge University Press.

[T] Talagrand M. (1996) Applying a theorem of Fernique, Ann. Inst. H. Poincaré, 32, p.779-799.

[W] Weber M. (1998) Une régularisation spectrale simplifiée, Publications de l'Université de Rennes, (3p.).

M.A.Lifshits, Komendantskii prospect, 22-2-49, 197372, St-Petersburg, Russia, email: lifts@mail.rcom.ru

M. Weber: Mathématique, Université Louis-Pasteur, 7, rue René Descartes, 67084 Strasbourg Cedex, France, e-mail weber@math.u-strasbg.fr 